\title{
Empty Wind Tunnel Flow Field Computations
}

\author{
Jean-Luc Hantrais-Gervois \\ ONERA, 8 rue des Vertugadins - 92190 Meudon - France \\ Aurélia Cartiéri \\ ONERA, 8 rue des Vertugadins - 92190 Meudon - France \\ Sylvain Mouton \\ ONERA, 5 boulevard Paul Painlevé - 59045 Lille Cedex - France \\ Jean-François Piat \\ ONERA, BP 25 - 73500 Modane - France
}

In this paper we try to introduce CFD to an unconventional place: the wind tunnel. We aim at gaining physical information concerning the wind tunnel flow field. The aim of this paper is to assess the capability to model the flow physics of an empty close wind tunnel. Detailed computations related to two major ONERA transonic wind tunnels are presented (S1MA and S2MA). The results of the different modelling of the wind tunnel flow field are presented and compared to experimental data.

\section{Nomenclature}

\begin{tabular}{|c|c|c|}
\hline S1MA & & $\begin{array}{l}\text { Large transonic wind tunnel in ONERA } \\
\text { Modane. The test section has a circular test } \\
\text { section ( } 8 \text { m diameter). }\end{array}$ \\
\hline S2MA & $=$ & $\begin{array}{l}\text { Pressurised transonic wind tunnel in ONERA } \\
\text { Modane. The test section has a rectangular } \\
\text { test section } 1.770 \mathrm{~m} \text { (height) } \times 1.750 \mathrm{~m} \\
\text { (width). }\end{array}$ \\
\hline CFD & $=$ & Computational Fluid Dynamics \\
\hline$C p$ & $=$ & Pressure coefficient \\
\hline$g$ & $=$ & Gravitational acceleration \\
\hline$H$ & $=$ & Height of a wind tunnel test section \\
\hline$M$ & $=$ & $\begin{array}{l}\text { Mach number. When the wall signatures are } \\
\text { concerned, Mach number is extrapolated to a } \\
\text { wall using the wall pressure and supposing an } \\
\text { isentropic flow. This figure has no reality } \\
\text { because the real wall velocity is zero. } \\
\text { Nevertheless it helps understanding the flow } \\
\text { evolutions in a wind tunnel better than the } \\
\text { static pressure, because it removes the total } \\
\text { pressure dependence. }\end{array}$ \\
\hline RANS & $=$ & Reynolds-Averaged Navier-Stokes \\
\hline $\operatorname{Re}$ & & $\begin{array}{l}\text { Reynolds number based on the aerodynamic } \\
\text { mean chord }\end{array}$ \\
\hline$x$ & $=$ & Distance along tunnel axis \\
\hline$V G$ & $=$ & Vortex generator \\
\hline$W$ & $=$ & Width of a wind tunnel test section \\
\hline WTT & $=$ & Wind tunnel test \\
\hline
\end{tabular}

\section{Introduction}

For more than a century, aerodynamics has been studied experimentally thanks to the rule that observing the still flow perturbed by a moving object is equivalent to observing a moving flow around a still object. Many wind tunnels have been built around the world; each being dedicated to specific flow regimes (Mach number, Reynolds number, turbulence levels, acoustics...). Moreover, a lot of models at a reduced scale have been tested in these facilities to provide aerodynamic data to the aircraft industry.

\section{A. Wind tunnel interference}

Aerodynamic differences between the model and the aircraft partly stem from an imperfect geometrical similarity of the model and from a different Reynolds number (except in cryogenic wind tunnels). They result also for a great part from the fact that the model is installed in a circuit; the associated flow disturbances arising from:

- wind tunnel walls (solid walls, ventilated walls or open tests sections);

- mounting systems (sectors, stings and struts, probes);

- $\quad$ history of the air circuit (fan, turns, honey combs, filters);

- $\quad$ flow turbulence, noise;

- moisture condensation.

Some of these effects stem from wind tunnel inherent flow imperfections and some other stem from the principle of testing a model in an air circuit. Except for wall effects, they can be partly measured (static pressure on the test section axis, flow deviations at some positions...); however the flow quality may not be completely known, even in extensively used wind tunnels.

Some basic requirements arise from the expected level of accuracy of the aircraft performance measurements, which is directly related either to commercial, safety or regulatory issues. International safety and regulatory requirements impose mandatory margins for normal operations with respect to the boundary of the flight domain (e.g. lift margin with respect to stall or buffeting). Accurate knowledge of 
this boundary is then required. As for commercial requirements, they mainly concern drag measurements at a given lift and Mach number, since it will directly impact fuel consumption. Pitching moment also enters this equation since it plays a role in additional drag to trim the aircraft. As indicated by Goldhammer1], the requirements from industry for the wind tunnel experiments concern:

- correctability: the flow around the model must not be modified in such a way that it can't be related to the ideal unbounded case through corrections (aerodynamic conditions and corrections to forces and moments);

- accuracy: the procedures used to correct the flow quantities must grant a known level accuracy.

It is important to draw a distinction between on the one hand the accurate knowledge of flow quantities at some point of the empty test section and the spatial (flow inhomogeneity) and temporal (flow unsteadiness) gradient of this quantity. The former is related to the quality of the measurement method and sensors, and not to the quality of the flow itself. On the contrary the later represents unwanted distortions of what should be a perfectly steady and homogeneous flow field.

Usual requirements in terms of measurement accuracy for longitudinal testing are:

- Up or downwash flow angle: an homogeneous flow angle with respect to the geometrical axis of the tunnel is corrected by testing the model upsidedown for a set of flow conditions. Therefore, this figure should not compromise the testing accuracy. However, large values of flow angle on the centreline of the test section are an indication of distorted flow which is likely not to be homogeneous. Once corrected, this figure should not exceed $\pm 0.1^{\circ}$.

- Mach number: \pm 0.001 is necessary to meet the $110^{-4}$ required drag accuracy below drag divergence together with a gradient of Mach number: \pm 0.001 over the length of the model.

Most wind tunnels, even among the modern ones, do not completely fulfil these requirements. Instead, they bring corrections to the produced data so as to account for their known limitations in flow quality. Hence, the most important matter might not be to achieve a perfectly homogeneous flow-field in the tunnel, which is very difficult at high Mach number, but rather to be aware of the limitations and to able to correct them properly. It seems highly desirable that the flow quality should be regularly controlled during the tunnel operating life, especially after repair or improvement works in the tunnel.

Any uncorrected inaccuracy beyond the above values, either detected from flow quality assessment or inferred from previous experience, obliges the aircraft manufacturer to take design margin with respect to the objective, which generally translates into cost penalties.

Furthermore, detailed experimental analyses of the flow field within a wind tunnel are rarely available. Such investigations are indeed difficult to perform because the distortions to measure are extremely small, so that introducing a probe in the area to investigate generates distortions that are much larger and should be carefully corrected. Thus, the flow quality may not be completely known, even in extensively used wind tunnels.

\section{B. CFD for the wind tunnel}

CFD is another means to provide data to the aircraft industry. It has also its drawbacks and inaccuracies but it allows obtaining data in the whole computed flow field. This is the reason why much can be gained through a detailed numerical analysis of the wind tunnel flow fields.

Many attempts to compute a wind tunnel flow field have been carried out. The very exhaustive and precious AGARDOgraph 336[2] collects and reviews all the numerical methods used until 1998 to account for the wind tunnel walls. Since this document, RANS CFD has become much more affordable and several attempts to compute the flow within a wind tunnel have been carried out. Bosnyakov et al[3], for example, have tackled the numerical modelling of the complex ETW wind tunnel. This facility features slots on the top and bottom walls which physics is discussed. Interesting results are presented on a model within the wind tunnel and in unconfined environment.

The modelling of the wind tunnel interference is also a subject of the Eurolift II4] European project and the German ForMEx5] program.

In this paper we present a detailed investigation of the ability of RANS CFD to model a wind tunnel flow, starting with the empty wind tunnel flow field.

Computing a wind tunnel is not the dedicated task of CFD that is not used to tackle huge geometries with machinery. The history of the air in the circuit is known to have a huge impact on the flow quality, but the complete modelling of this circuit is still beyond reach and is not tackled in this paper. Thus, the most relevant parts for the mounting and wall interferences have been modelled (convergent, test section mounting system, diffuser). Even if this portion could be viewed as a simple tube easy to compute by hand, reality is different:

- evolution of the boundary layer over the long distance considered together with the velocity gradients and wall curvature;

- $\quad$ the mounting system can be complicated (tripod);

- $\quad$ some flow features differ from a clean wall (steps, vortex generators...).

The paper will first describe the facilities of interest in this study. The numerical methods applied to compute the wind tunnel flow fields will then be presented. The validation of the empty tunnel flow computations against experimental results will then be presented with the analysis of several wind tunnel modelling effects:

- $\quad$ effect of the wind tunnel extension to model;

- $\quad$ mesh density effects (S1MA);

- modelling of the S1MA vortex generators;

- modelling of the S2MA re-entry flaps; 
- wall roughness (S2MA) and turbulence model effects (S1MA).

\section{Wind tunnels}

\section{The ONERA Modane-Avrieux centre}

One of the missions of the French public aerospace research centre (ONERA) is to provide technical assistance to worldwide aerospace industry and research teams, by operating several wind tunnels. Among the facilities, the Modane-Avrieux and Le Fauga-Mauzac test centres commercially operate a range of large, unique wind tunnels, spanning the entire speed range to hypersonics.

Among the Modane-Avrieux facilities (see Table 1 and Figure 1), the S1MA and S2MA wind tunnels have been chosen for this numerical application because of their intensive use for industrial purposes. The facilities differ by their sizes (S1MA exhibits a test section of about $45 \mathrm{~m}^{2}$, to be compared with $3.1 \mathrm{~m}^{2}$ for S2MA) but also by their conditions (S1MA is atmospheric whereas S2MA is pressurized). In this study we have focused on the subsonic and transonic regimes and we have considered the closed test sections for both wind tunnels (S2MA is designed to be operated with porous floor and ceiling).

\begin{tabular}{|c|c|c|c|}
\hline $\begin{array}{l}\text { Facility } \\
\text { name }\end{array}$ & $\begin{array}{l}\text { Facility } \\
\text { type }\end{array}$ & $\begin{array}{l}\text { Flow } \\
\text { regime }\end{array}$ & $\begin{array}{l}\text { Test section } \\
\text { geometry }\end{array}$ \\
\hline S1MA & $\begin{array}{l}\text { continuous } \\
\text { atmospheric }\end{array}$ & sub/transonic & $\begin{array}{l}\text { Close to circular } \\
\varnothing \text { about } 8 \mathrm{~m} \text { (several } \\
\text { carts available) }\end{array}$ \\
\hline S2MA & $\begin{array}{l}\text { continuous } \\
\text { pressurized }\end{array}$ & $\begin{array}{l}\text { sub/trans/ } \\
\text { supersonic }\end{array}$ & $\begin{array}{l}\text { rectangular } \\
1.75(\mathrm{~W}) \times 1.77(\mathrm{H}) \mathrm{m}^{2} \\
\text { (sub/transonic) } \\
1.75(\mathrm{~W}) \times 1.93(\mathrm{H}) \mathrm{m}^{2} \\
\text { (supersonic) }\end{array}$ \\
\hline S3MA & $\begin{array}{l}\text { blow-down } \\
\text { pressurized }\end{array}$ & $\begin{array}{l}\text { sub/trans/ } \\
\text { supersonic }\end{array}$ & $\begin{array}{l}\text { rectangular } \\
0.56(\mathrm{H}) \times 0.78(\mathrm{~W}) \mathrm{m}^{2} \\
\text { (sub/transonic) } \\
0.76(\mathrm{H}) \times 0.80(\mathrm{~W}) \mathrm{m}^{2} \\
\text { (supersonic) }\end{array}$ \\
\hline S4MA & blow-down & hypersonic & $\begin{array}{l}\text { circular } \\
\varnothing 0.68 \mathrm{~m} \text { to } 1.00 \mathrm{~m}\end{array}$ \\
\hline R4 & $\begin{array}{l}\text { cascade wind- } \\
\text { tunnel }\end{array}$ & $\begin{array}{l}\text { sub/trans/ } \\
\text { supersonic }\end{array}$ & $\begin{array}{l}2 \mathrm{D} \\
0.12 \mathrm{~m}(\mathrm{~W})\end{array}$ \\
\hline S4B & $\begin{array}{l}\text { model engine } \\
\text { calibration } \\
\text { facility } \\
\text { (vacuum } \\
\text { tank) } \\
\end{array}$ & & \\
\hline TURMA & $\begin{array}{l}\text { two turbine } \\
\text { stages test rig }\end{array}$ & & \\
\hline
\end{tabular}

Table 1: ONERA Modane facilities.

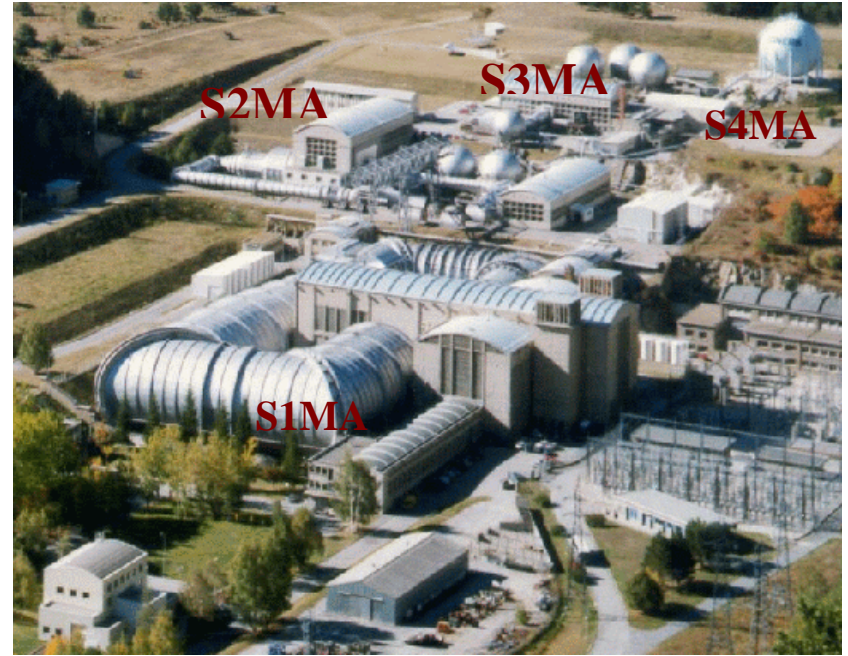

Figure 1: ONERA Modane-Avrieux centre and main wind tunnels.

\section{S1MA presentation}

S1MA is a continuous atmospheric wind tunnel operating in the sub/transonic regime. It was put into service in 1952 and is equipped with two contrarotating fans, driven by Pelton turbines, the power of which is $80 \mathrm{MW}$. The wind velocity can be varied from a few meters per second to approximately Mach 1 by varying the fan speed.

The total length of the aerodynamic circuit is about $400 \mathrm{~m}$. A peculiarity of the circuit is the absence of heat exchanger. The temperature is controlled by letting fresh outside air enter the circuit. Hot air naturally exhausts around the edge of the contraction through an annular exit. An exhaust rate of about $10 \%$ of the total mass flow is required to maintain a temperature of about $50^{\circ} \mathrm{C}$ in the tunnel (see Figure 4, left).

Among the three interchangeable carts available, cart number 2 has been used for this study. This test section features solid walls and a geometrical cross-section of about $45.0 \mathrm{~m}^{2}$. It is well suited to tests on elements of real aircraft and on large scale models (4 m span or half span).

\section{E. S2MA presentation}

S2MA is a continuous pressurized wind tunnel operating in the sub/trans/supersonic regime. This was put into service in 1961 and is driven by a 57 MW compressor powered by four Pelton turbines.

In this study, only the transonic test section has been considered (1.75 m width $\mathrm{x} 1.77 \mathrm{~m}$ height). In this test section the Mach number and the stagnation pressure can be varied from 0.1 to 1.3 and from 0.15 to a maximum of 2.5 bars respectively. The transonic test section is used for small scale models ( $1 \mathrm{~m}$ to $1.2 \mathrm{~m}$ span or half span) and for a lot of specific tests (air intakes, store separation, dynamic stability, buffeting and flutter ...).

This test section is mainly operated with perforated floor and ceiling (the average porosity amounts $2.9 \%$ ). In this study we have only tackled the closed test section which is not the usual setting for this wind tunnel; this was achieved by 
sealing the horizontal porous walls. Nevertheless, a communication between the plenum and the test section still exists aft the test section, upstream to the sector (small steps indicated as walls in Figure 4, right)

Since the test section had been designed to minimize the wall interference through porous walls, it features no divergence and a longitudinal velocity gradient is present inside this unconventional closed test section.

\section{Numerical methods}

\section{A. CFD software}

We have resorted to RANS computations using the els $\mathrm{A}^{6,7}$ software. The software solves the equations in a finite volume formulation (cell-centred). For this study, a LU implicit scheme has been used together with a second order central difference for the spatial discretisation of the mean flow (Jameson scheme with second and fourth order dissipation terms). As for the turbulent quantities, a first order central discretisation is used. The time scheme is the first order backward-Euler. The local time stepping and multigrid techniques are applied to speed up the convergence process.

The computations resort to RANS modelling computed with the elsA[6], [7] software. Structured meshes have been built on the wind tunnel geometries. The size of the meshes is part of the study (see next chapter, section 2). Due to the geometric complexity of the elements present in the wind tunnels (tripod, sector, vortex generators...), meshing techniques like Chimera and non coincident joints have been used to ease the topology of the blocking (see Figure 3).

As for turbulence modelling, various approaches have been tested and will be detailed in next chapter sections 5 and 6 . Whereas the S2MA wind tunnel has been computed with low Reynolds turbulence model, the S1MA wind tunnel has been computed with wall law approach to spare boundary layer mesh points.

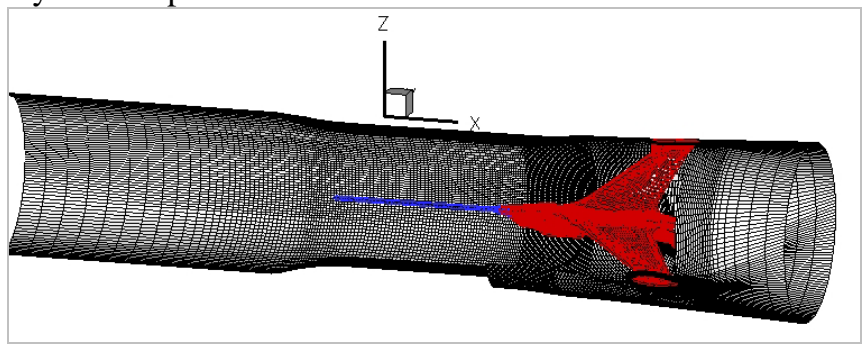

Figure 2: S1MA mesh, the tripod is in red and the calibration probe in blue.

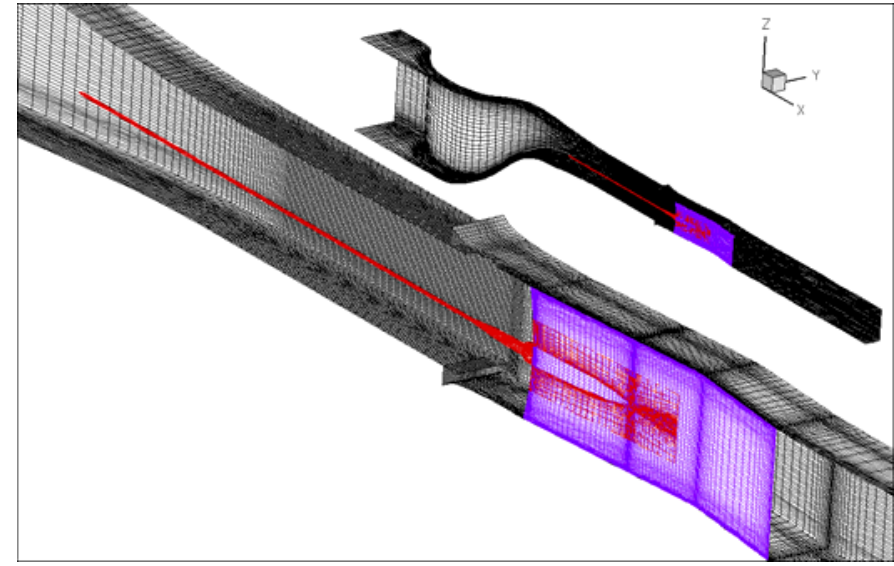

Figure 3: S2MA mesh (half configuration), the probe (red) is added on top the empty test section with the sector (black and purple) through the Chimera technique.

\section{B. Wind tunnel CAD}

For both wind tunnels, the complete air circuit is difficult to account. The geometrical details together with the physics involved in the honey combs, turns... is beyond the scope of this study. Thus, we have chosen to keep the part where most of the aerodynamic phenomena take place (see Figure 4):

- $\quad$ settling chamber;

- convergent;

- test section;

- divergent.

In this model, the history of the fluid can't be reproduced, but the Mach number gradients and boundary layers in the test section are modelled.

Since the flow becomes more an more sensitive to geometry flaws when its velocity increases in the transonic range, the accuracy of the CAD model in high speed areas of the tunnel must be known with good accuracy. We retained the objective of modelling the Mach number and the flow angle in the test section to accuracy respectively better than 0.001 and $0.01^{\circ}$ and Mach numbers up to 0.95 (objective better than required for industrial applications). By simulating the inviscid transonic flow in an ideal circular wind tunnel in which a bump is introduced, it was found that these requirements were fulfilled near the centre of the test section if the bump spreads over less than $20^{\circ}$ azimuth, has a height and a length respectively less than 0.001 and 0.25 radius of the section. This provided guidelines to determine whether the existing CAD model were accurate enough. As for S2MA, the layout of the construction of the tunnel ensured sufficient accuracy compared to the drawings.

In the case of S1MA, because of the age of the tunnel cart (1952, modified several times) and because of the construction type (welded steel panels), the surface of the test section is not smooth. Therefore, in situ measurements of the shape of the test section were carried out to acquire 1416 positions of points to an accuracy better than $0.01 \mathrm{~mm}$. A new CAD model of the test section was then built based on this dataset. 

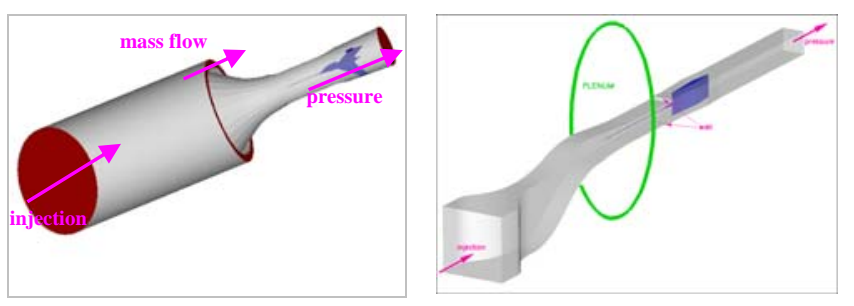

Figure 4: S1MA (left) and S2MA (right) CAD models for CFD applications.

\section{Boundary conditions}

For the modelled wind tunnels, apart from walls, the boundary conditions consist of the inlet (settling chamber) and the outlet (part of the diffuser). The inlet is imposed as a reservoir condition: stagnation pressure and stagnation temperature are imposed; speed direction is supposed to be lined up with the tunnel axis (four entering characteristics). The norm of the speed is not imposed and is part of the converged solution (fifth characteristic). The turbulence rate is imposed as $0.05 \%$ in S1MA. The S2MA computations did not use this quantity as the Spalart-Allmaras model used for this study does not use it. For both wind tunnels, the turbulent viscosity ratio of $0.1 \%$ has been used. These values do not relate to the physical values of the wind tunnels.

As for the outlet, only one flow quantity needs to be prescribed. Consistently with the physics of the flow in a pipe, a constant pressure is imposed. From this pressure depends the mass flow circulating in the wind tunnel, and therefore the Mach number in the test section. The outlet pressure is adjusted to retrieve a required Mach number in the test section. This procedure is common for wind tunnel computations (see for example Bosnyakov et al[3]). Automatic procedures have been developed and used to iterate on the outlet pressure so as to achieve a target wind tunnel regime.

As for S1MA, the annular hot air exhaust around the start of the contraction was given a target mass-flow boundary condition. The solver iterates until the rate of mass flowing through that boundary condition reaches the prescribed value, which is given as a percentage of the total mass flow expected to circulate in the tunnel at the target Mach number. In S2MA, the modelling of the re-entry flaps connecting the test section and the plenum has been studied in section 4 .

\section{Empty tunnel results}

The aim of this part was to select the sufficient models to retrieve the sufficient flow features for our purposes. As we aim at being able to compute wind tunnel wall interference, the main issue is to be able to represent:

- The velocity gradients along the test section, including what is generated by the permanent part of the model support system (tripod in S1MA, sector in S2MA)

- The flow angularity (incidence and sideslip)
These requirements imply that the growth of the boundary layers is correctly predicted along the wind tunnel walls. As for flow angularity, the history of the air circuit can have an impact and the model does not take this into account, which is certainly one of the largest limitations of our simulations.

Several aspects of the wind tunnel modelling are investigated and modelling guidelines are identified. The validation is carried out with a validation against experimental calibration results and boundary layer measurements. The wall pressure measurements are strongly linked to the waviness of the walls. Thus, on a longitudinal wall pressure curve, oscillations can occur. It is usual to smooth these oscillations when comparing with CFD, but the rough values where used in this paper. These oscillations are local and the centreline pressure distribution is smooth.

As for S1MA, mesh density effect and turbulence modelling and shortening effects are all studied on a configuration without tripod inside the wind tunnel (for convergence issues).

\section{Geometrical modelling}

\section{Vortex Generators in S1MA}

A calculation including the vortex generators (VGs) has been performed. The computational domain includes the settling chamber, the convergent, the test section and the entire diffuser (see Figure 5). A comparison without the VGs is also performed. The efficiency of the VGs is revealed: the large separation zone that appears (at about $\mathrm{X}=30 \mathrm{~m}$ ) on the configuration without the VGs is then suppressed when including them (Figure 6).

For convergence issues, it has thus been decided to stop the computational domain around $\mathrm{X}=25 \mathrm{~m}$ in the diffuser so as not to model the VGs.

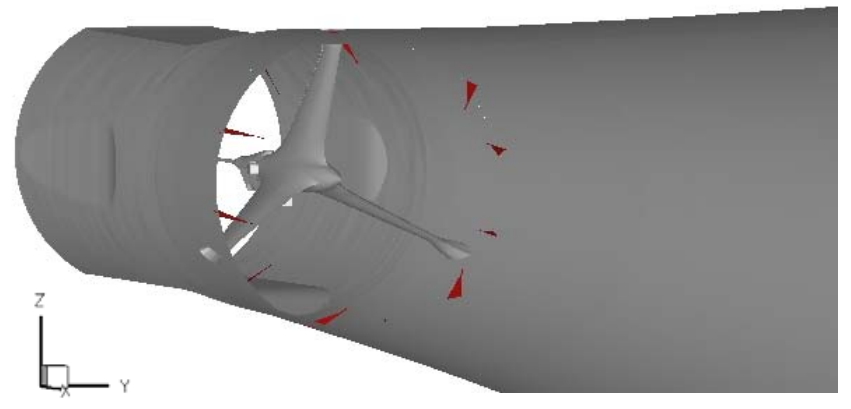

Figure 5: View of the Vortex Generators (VGs) in S1MA wind tunnel.

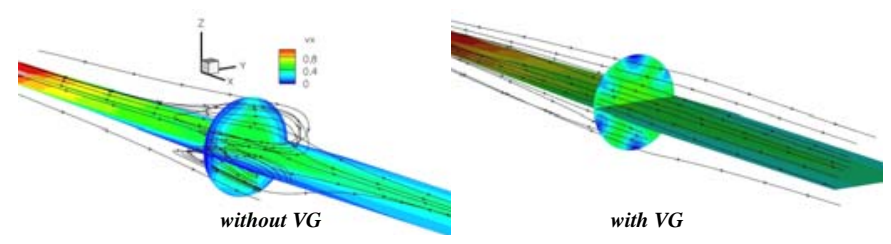

Figure 6: Effect of the VGs on the flow field. 


\section{Mesh density effect S1MA}

In order to reduce the number of mesh points to lower the CPU time, the mesh density effect in axial direction is studied. The following meshes have been tested on 3 different Mach numbers: 0.205, 0.801 and 0.848 (see Table 2).

\begin{tabular}{|c|c|c|c|c|}
\hline Mesh identification & $\mathbf{1}$ & $\mathbf{2}$ & $\mathbf{3}$ & $\mathbf{4}$ \\
\hline $\begin{array}{c}\text { Total number of points } \\
(\mathbf{x 1 0} \text { ) }\end{array}$ & 3.0 & 2 & 1.7 & 1.2 \\
\hline $\begin{array}{c}\text { Axial distance between } \mathbf{2} \\
\text { point in test section (in cm) }\end{array}$ & 10 & 17 & 21 & 26 \\
\hline
\end{tabular}

Table 2: S1MA mesh refinement study - mesh characteristics.

No mesh effect can be observed on the boundary layer profiles for the three Mach Numbers.

As for wall signatures, both experiments and numerical simulations exhibit oscillations at the wall (see Figure 7). These oscillations in wall Mach number - amounting about \pm 0.01 - stem from the wall waviness and are localised near the wall. In the model area, Mach number variations do not exceed $2.10^{-4}$. Figure 7 shows that the oscillations are slightly reduced as the axial discretisation decreases. Up to a Mach number of 0.85, the largest mesh leads to very good results. But in presence of shocks on the wind tunnel walls, the results are highly sensitive to axial discretisation. The effects of a high Mach number on wall signatures are shown in Figure 7. The oscillations have moved forward and their amplitude is also lowered. In fact, the shocks are not correctly captured with the coarser meshes. As a conclusion, it might be necessary to have a rather fine mesh when high Mach numbers are targeted.
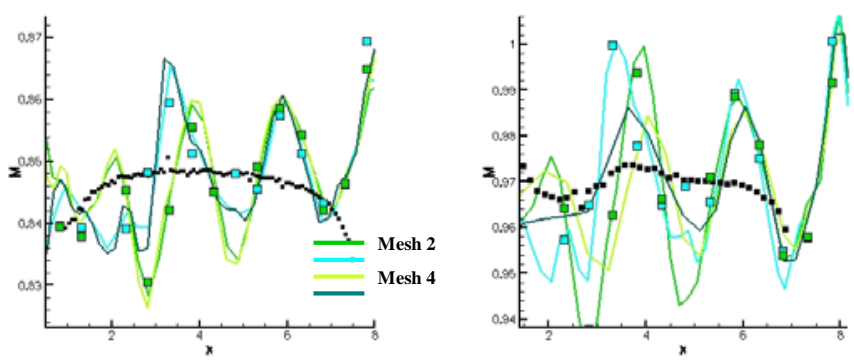

Figure 7: Wall signatures (Mesh 2 and Mesh 4) for an usual Mach number (left) and a high Mach number (right), tunnel Centerline in black symbols.

\section{Extension of the geometry to consider (S1MA and S2MA)}

For both wind tunnels, the effect of shortening the wind tunnel portion to take into account has been investigated. The aim is to reduce the complexity of the computation, removing low speed flows impeding the numerical rapidity and also to reduce the number of mesh points to lower the CPU time.

As for S1MA, the shortening corresponds to Figure 8. The convergent is suppressed and a certain length of cylindrical wall is added so as to ensure a correct boundary layer profile at test section entry and a length of inviscid wall for boundary conditions purpose. The CPU time have thus been lowered.

The calculations are performed without tripod.

Both boundary layer profile and tunnel centreline are still well predicted (see Figure 9). Moreover, the flow angularity is quite the same in an axial plane inside the test section despite the convergent was suppressed (see Figure 10). No precision was lost which can enable us to use this shortened case.

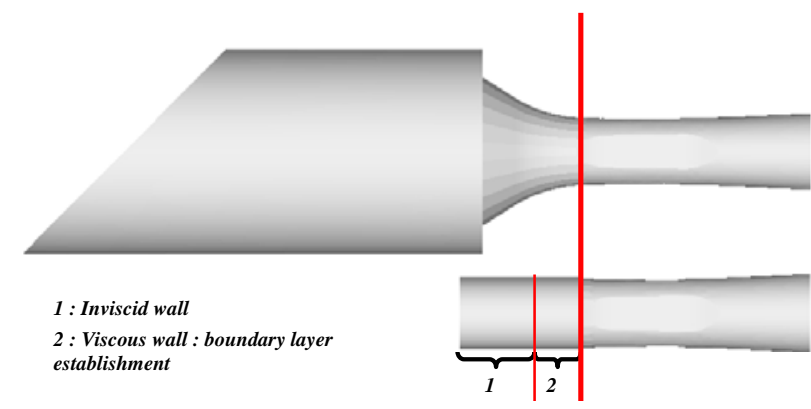

Figure 8: S1MA geometric shortening - complete test section (up) and shortened (down).

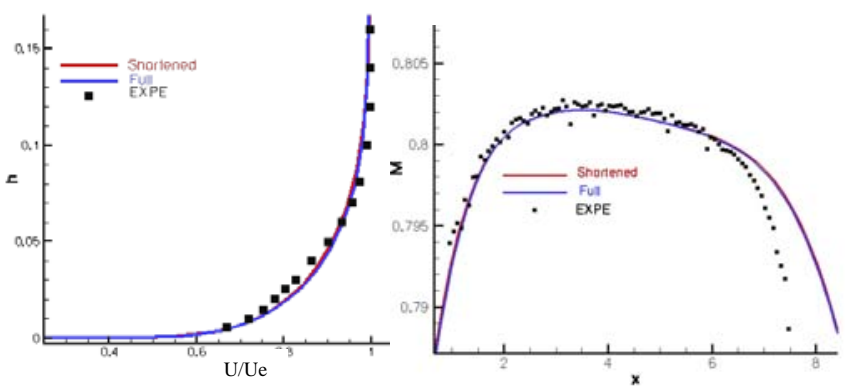

Figure 9: Boundary layer profile (left) and tunnel centreline (right).
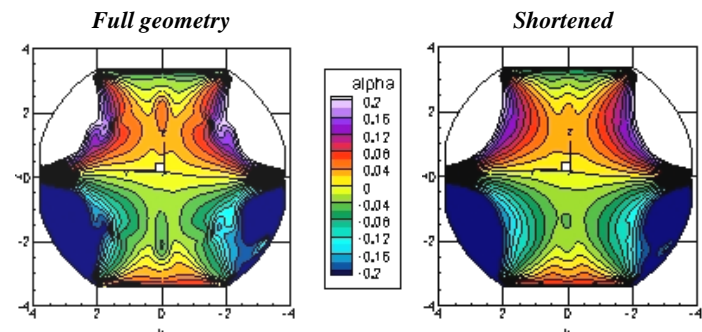

Figure 10: Incidence distribution in the wind tunnel.

As for S2MA, the shortening corresponds to Figure 11. The diffuser is suppressed, together with the convergent. Ahead of the test section, a certain length of wall is added in order to retrieve a correct boundary layer thickness at the test section inlet. The computations have been dramatically accelerated by the shortening. Nevertheless, the velocity gradient along the tunnel centreline lost precision (see Figure 12).

Thus, contrary to S1MA, the S2MA convergent and diffuser need to be modelled. 

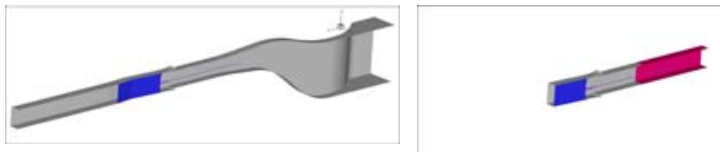

Figure 11: S2MA geometric shortening - complete (left) and shortened (right).

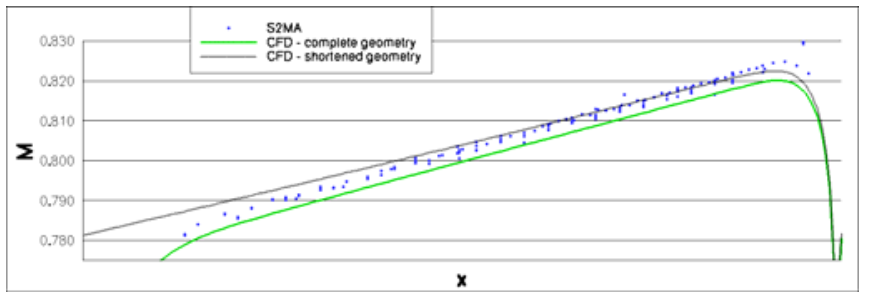

Figure 12: Effect of the shortening of the geometry on the gradient in the test section.

\section{Re-entry flap modelling in the S2MA wind tunnel}

Even when the S2MA porous walls are sealed, the re-entry flaps allowing air to circulate between the plenum and the test section are open. The mass flow in this region is not well known and only the plenum pressure is measured during a test. The numerical problem is then ill-conditionned for flow entering the test section.

We have tried to model this re-entry flap through several ways presented in Figure 13:

1. communication between the plenum and the test section (pressure condition);

2. smoothed flap;

3. closed flap.

The first model has difficulties to converge because the air exchange in the flap region depends on the pressure inside the wind tunnel. As the pressure evolves with the computation convergence, the type of flow in this area can swap form exiting flows to entering flows. A correct convergence could only be achieved when starting the computation to an already converged case with a closed flap. The other two cases converge correctly.

The results as far as pressure gradient is concerned are presented in Figure 14. All models provide correct gradients in the test section. Nevertheless, the first model diverges from the other aft the test section and the wall pressures are wrong in the diffuser. Difficulties with this model would be encountered when high Mach numbers will be computed because shocks on the sector will arise.

The closed re-entry flap model provides the best results all along the walls and has thus been kept for the other computations.
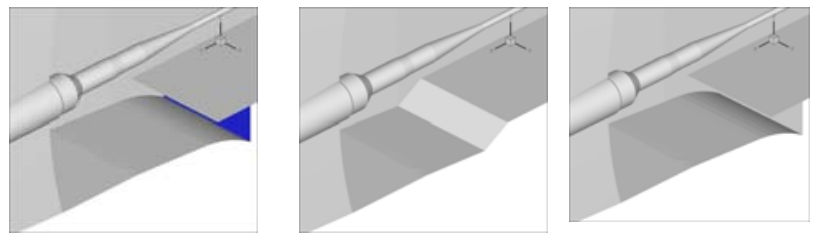

Figure 13: Re-entry flap models - communication with the plenum (left), smoothed step (centre) and closed flap (right).

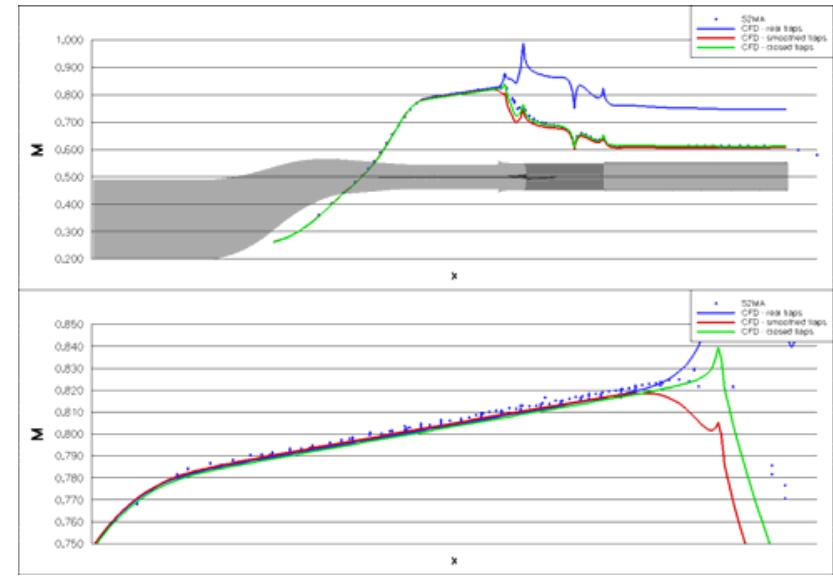

Figure 14: Re-entry flap modelling effect in S2MA - full wind tunnel (up) and test section close up (down).

\section{E. Numerical modelling}

\section{Turbulence model effect S1MA}

As the main issue is to compute wind tunnel wall interference, we might want to choose the turbulence model according to the aircraft model without being bothered by wall effects. Several models for turbulence closure are therefore investigated:

- Spalart-Allmaras;

- K- $\omega$ of Wilcox (with Zheng Limiter) with SST correction;

- K- $\omega$ of Menter (with Zheng Limiter) with SST correction;

- K-l of Smith;

- $\quad$ two-layers k- $\varepsilon$ of Launder \& Sharma.

Except for the k-l model of Smith, all models have good results both in boundary layer and tunnel centreline Mach number (see Figure 15). In particularly, most currently used models k- $\omega$ and Spalart-Allmaras give good results.

The prediction of Mach number distribution on centreline is very good (except for model k-l of Smith). The lack of deceleration on the computations beyond $\mathrm{x}=7 \mathrm{~m}$ is due to tripod which is not simulated on these sensibilities.

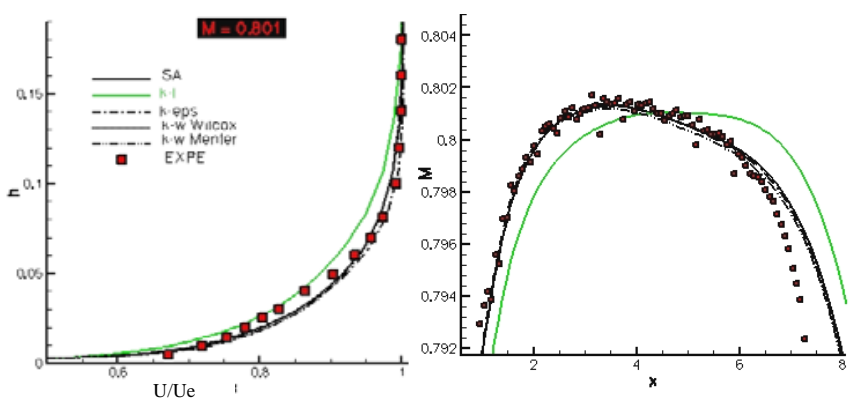

Figure 15: Boundary layer profile (left) and tunnel centreline (right).

\section{Wind tunnel wall roughness effect S2MA}

As the gradient obtained in the test section without roughness was slightly lower than the experimental gradient, 
it indicated a development of boundary layers slightly underestimated over the test section. Thus the elsA model for wall roughness has been used to improve this aspect. This roughness corresponds to a sand grain thickness. The height of the grain has been varied from $0 \mathrm{~mm}$ to $0.1 \mathrm{~mm}$ (see Figure 16). A great non linear effect is sensed and a fine tuning of the roughness height is required. Slight modifications of the height lead to important variations of the gradient.

Based on a low total pressure, a value has been selected for the roughness height. Nevertheless, this same value leads to an inverse total pressure effect with total pressure when the absence of roughness provides a correct effect (the gradient experimentally diminishes with total pressure, see Figure 17). It has thus been decided to avoid the use of wall roughness for the study, especially in S2MA that covers large total pressures.

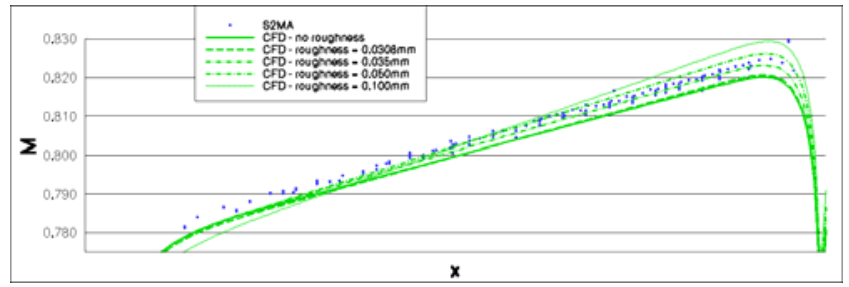

Figure 16: Roughness effect on the gradient in the test section low total pressure.

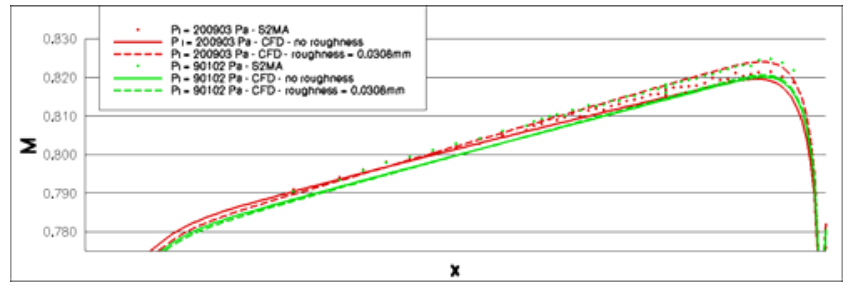

Figure 17: Roughness effect on the gradient in the test section low and high total pressures.

\section{F. Final wind tunnel models selected}

Some specific wind tunnel features have been tackled. As for S1MA, the vortex generators could be removed by simply cutting the geometric model of the wind tunnel upstream to the VGs, without any modification of the test section flow. The S1MA geometry with a shortened convergent has been selected, whereas the whole S2MA convergent had to be kept. In S2MA, the re-entry flaps have finally been modelled as closed, with a numerical robustness gain and without any accuracy loss.

The Spalart-Allmaras turbulence model without any roughness model provides correct results for both wind tunnels and several other turbulence models have been validated in S1MA.

The features of the meshes finally selected are presented in Table 3. About 3 to 4 million nodes are used for both wind tunnels. This relatively moderate figure spares some mesh points for further applications involving a model enclosed in these models.

\begin{tabular}{|c|c|c|c|c|}
\hline & \multirow{2}{*}{$\begin{array}{l}\quad \text { S1MA } \\
\text { Shortened } \\
\text { Wind } \\
\text { Tunnel } \\
\text { tripod }\end{array}$} & \multicolumn{2}{|c|}{ S2MA } \\
\hline & & & $\begin{array}{l}\text { Wind } \\
\text { tunnel }+ \\
\text { sector }\end{array}$ & $\begin{array}{l}\text { Probe }+ \\
\text { sting } \\
\text { boss }\end{array}$ \\
\hline $\begin{array}{l}\text { Total number of } \\
\text { points }\end{array}$ & & $3,409,568$ & $3,134,881$ & $1,159,557$ \\
\hline $\begin{array}{l}\text { Total number of } \\
\text { cells }\end{array}$ & & $3,182,272$ & $3,069,824$ & $1,115,520$ \\
\hline $\begin{array}{l}\text { Number of } \\
\text { blocks }\end{array}$ & & 48 & 7 & 14 \\
\hline $\begin{array}{l}\text { Number of } \\
\text { multigrid levels }\end{array}$ & & 3 & 3 & 3 \\
\hline \multirow{3}{*}{$\begin{array}{l}\text { Mesh size in the } \\
\text { test section }\end{array}$} & longitudinal & $190 \mathrm{~mm}$ & $40 \mathrm{~mm}$ & $60 \mathrm{~mm}$ \\
\hline & lateral & $80 \mathrm{~mm}$ & $20 \mathrm{~mm}$ & $10 \mathrm{~mm}$ \\
\hline & vertical & $130 \mathrm{~mm}$ & $40 \mathrm{~mm}$ & $25 \mathrm{~mm}$ \\
\hline \multicolumn{2}{|l|}{$\begin{array}{l}\text { First cell size in } \\
\text { the test section }\end{array}$} & $0.1 \mathrm{~mm}$ & $\begin{array}{r}0.0025 \\
\mathrm{~mm} \\
\end{array}$ & $\begin{array}{r}0.0025 \\
\mathrm{~mm} \\
\end{array}$ \\
\hline \multicolumn{2}{|l|}{$\begin{array}{l}\text { Corresponding } \\
y^{+} \text {value in the } \\
\text { test section }\end{array}$} & $\begin{array}{lr}\text { about } 50 & \text { with } \\
\text { wall } & \text { law } \\
\text { approach } & \end{array}$ & $\begin{array}{l}\text { about } 0.7 \\
\text { to } 2 \text {, } \\
\text { depending } \\
\text { on the } \\
\text { total } \\
\text { pressure }\end{array}$ & $\begin{array}{l}\text { about } 0.7 \\
\text { to } 2 \text {, } \\
\text { depending } \\
\text { on the } \\
\text { total } \\
\text { pressure }\end{array}$ \\
\hline
\end{tabular}

Table 3: Mesh characteristics.

\section{G. Validation of the flow field}

Based on the elements selected in previous chapter, we will now focus on the detailed validation of the computations. The validation is carried out through a comparison with wind tunnel measurements for several Mach numbers and stagnation pressures (wall signatures, boundary layer profiles and clinometric measurements).
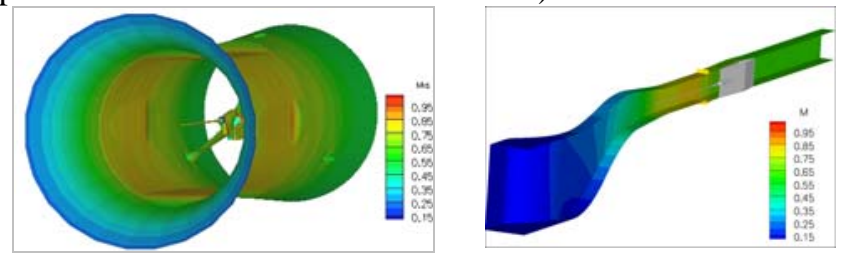

Figure 18: Example of isentropic Mach number evolution in the empty closed wind tunnels $(M=0.8)$ - S1MA (left) and S2MA (right).

\section{Boundary layer profiles}

As for S1MA, several boundary layers measurements are available:

- $\quad$ in entry test section (section 1);

- $\quad$ in the middle and the end of test section (section 2$3)$.

The results in some sections are given below (Figure 19).

The agreement is rather good on section presented. The boundary layer thickness is well predicted. 

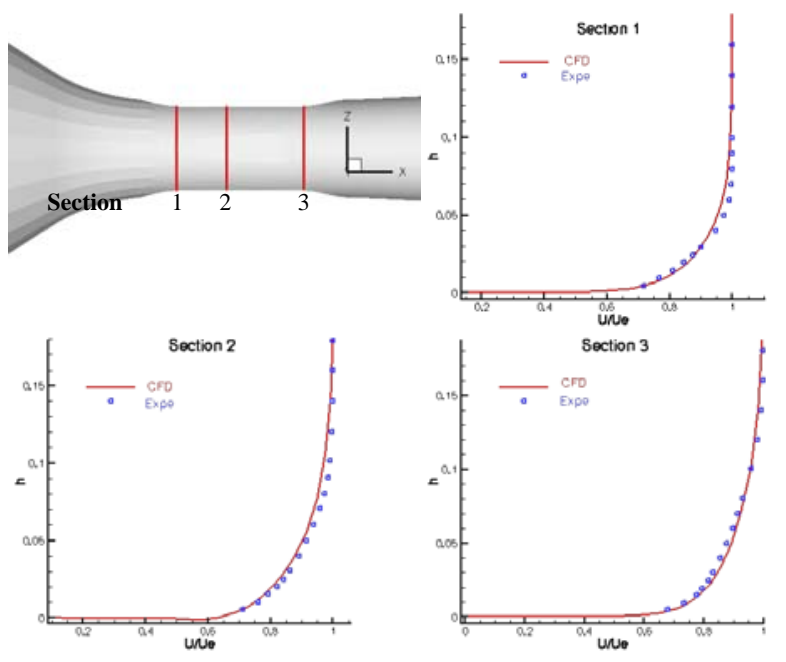

Figure 19: Boundary layer profiles in 3 different S1MA sections, CFD in solid line, experiments in symbols.

As for S2MA, the boundary layer data available for the closed test section include a model in the wind tunnel. Thus these data must only be taken as indicative. In Figure 20, all the model lift coefficients are plotted together showing a kind of error band. The computations indicate a small total pressure effect that is not seen in the experiments due to the error band. As in the S1MA case, the results are correct.

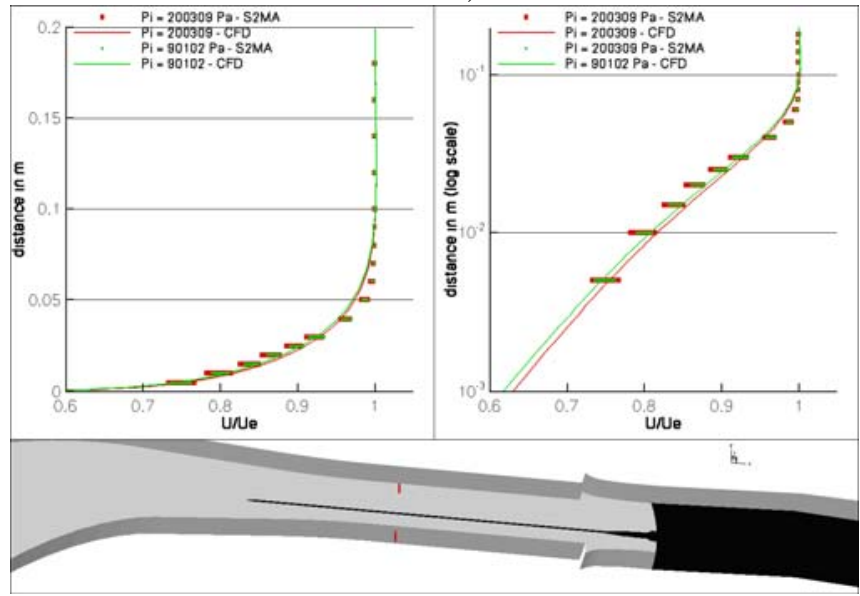

Figure 20: S2MA boundary layer profiles - CFD in solid lines and experiments in symbols $(M=0.8)$. The red lines in the S2MA indicate the location of the boundary layer rakes.

\section{Wind tunnel calibrations}

As for S1MA, here are some results with tripod inside the wind tunnel (Figure 21). The prediction on tunnel centreline is excellent even beyond $\mathrm{x}=7 \mathrm{~m}$. As for wall signatures, the agreement with experimental results is also very good. The oscillations are well captured by the computations (Figure 22).
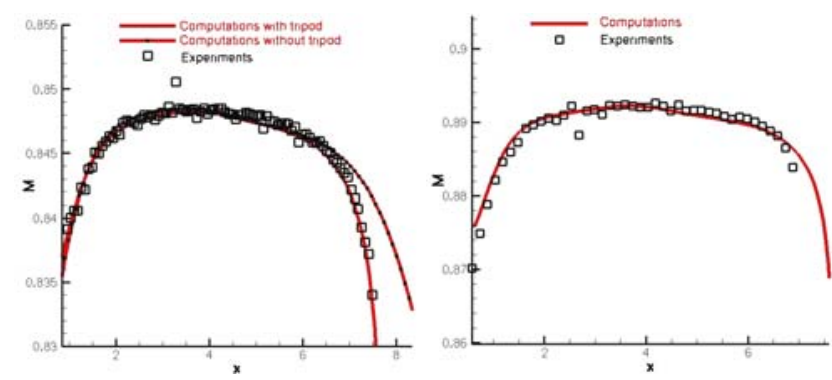

Figure 21: Tunnel centreline for $M=0.85$ (left) and $M=0.89$ (right), CFD in solid line, experiments in symbols.
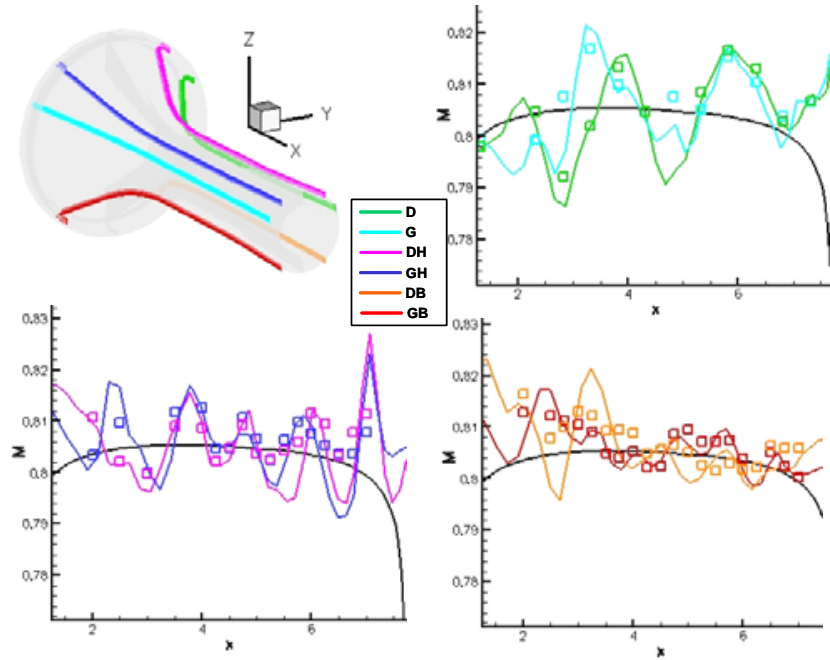

Figure 22: Wall isentropic Mach number distribution in test section for $M=0.8, C F D$ in solid line, experiments in symbols.

As the S2MA wind tunnel is pressurised, it is necessary to cover a matrix of computations to validate the behaviour of the computation against the experimental range. The matrix covered is:

- $\mathrm{M} \in[0.7,0.9]$;

- $\quad \mathrm{Pi} \in[0.9$ bar, 2.0 bars].

The computed wall and probe pressures are compared to the experimental ones in Figure 23. We see an excellent overall agreement at this scale. In order to be more precise, the numeric value of the test section Mach number gradient is also plotted in Figure 24. The agreement is very good up to $\mathrm{M}=0.875$ and the total pressure effects are very well accounted for. At $M=0.9$, a discrepancy appears.

These results validate the modelling used for S2MA. 


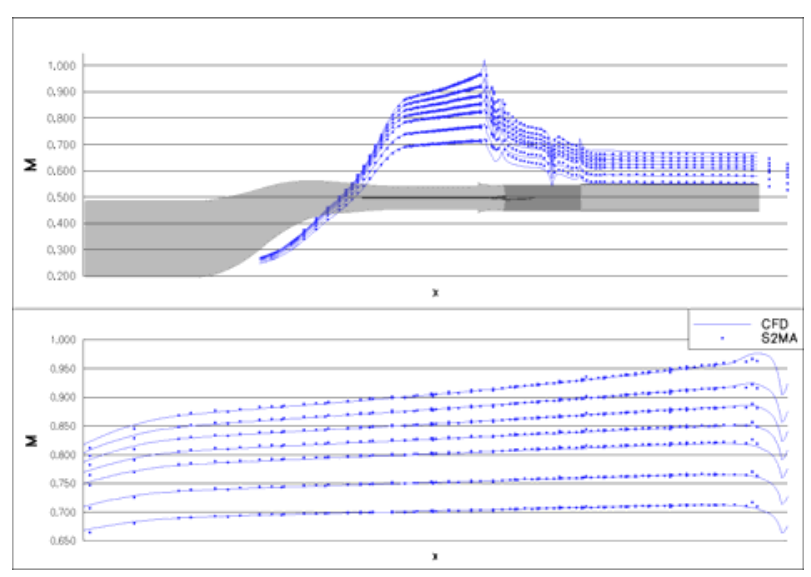

Figure 23: Comparison of the wall signatures in S2MA for several Mach numbers about $\mathrm{Pi}=2$ bars - full wind tunnel (up) and test section close up (down).

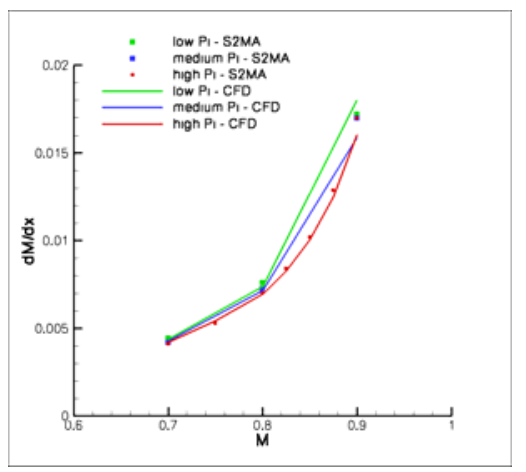

Figure 24: Validation of the longitudinal gradients in the S2MA test section.

\section{Conclusions}

In this paper, we have thoroughly investigated the ability of RANS equations and solver to model a flow field within empty wind tunnels, focusing on the determination of attainable accuracy at low computational cost. At first sight, computing an empty tube does not seem to be of any interest for these expensive computing tools. However, computations confirmed or revealed interesting features of the flow non-uniformities. Moreover, similar additional computations were carried out to assess the effect in the centre of the test section of different geometrical or aerodynamic flows that can be easily introduced in the model (e.g. asymmetric mass-flow rate in annular exhaust of S1MA).

We have thus brought our conclusions on the geometry to model, the numerical model for the walls and for turbulence and the means to operate the computations. The results achieved correlate correctly with the experiments as far as boundary layer and velocity gradients are concerned.

\section{Acknowledgments}

The authors would like to thank the French Government for financing this research.

\section{References}

1. Goldhammer, M. I., Wall Interference, Support Interference and Flow Field Measurements - Technical Evaluation Report, AGARD Advisory Group for Aerospace Research and Development, 1994, AGARD-CP-535.

2. Ashill, P. R.; Binion, T.; Cooper, K. R.; Crites, R.; Everhart, J. L.; Ewald, B. F.; Hackett, J.; Holst, H.; Krynytzky, A. J.; Malmuth, N. D.; Mokry, M.; Newman, P. A.; Sickles, W. L.; Steilne, F. W. J.; Taylor, C. R.; Taylor, N. J.; Voss, R. \& Wedemeyer, E. H., Wind Tunnel Wall Corrections, AGARD Advisory Group for Aerospace Research and Development, 1998, AGARD-AG-336.

3. Bosnyakov, S.; Kursakov, I.; Lysenkov, A.; Matyash, S.; Mikhailov, S.; Vlasenko, V. \& Quest, J., Computational tools for supporting the testing of civil aircraft configurations in wind tunnels, Progress in Aerospace Sciences, 2008, 44, pp 67-120.

4. Eliasson, P., Numerical validation of a half model high lift configuration in a wind tunnel, 45th AIAA Aerospace Sciences Meeting and Exhibit, AIAA, 2007, AIAA 2007262, AIAA 2007-262.

5. Melber-Wilkending, S.; Heidebrecht, A. \& Wichmann, G. ICAS (ed.) A New approach in CFD supported wind tunnel testing 25th International Congress of the Aeronautical Sciences, ICAS, 2006, ICAS 2006-3.4.2, ICAS 2006-3.4.2.

6. Cambier, L., Gazaix, M., elsA: an Efficient Object-Oriented Solution to CFD Complexity, AIAA Aerospace Sciences Meeting and Exhibit, Reno, Jan. 2002.

7. Cambier, L., Veuillot, J.-P., Status of the elsA Software for Flow Simulation and Multi-Disciplinary Applications, 46th AIAA Aerospace Sciences Meeting and Exhibit, Reno, Nevada, Jan. 7-10, 2008. 